\title{
Measuring gluon sivers function at a future Electron-Ion Collider
}

\author{
Liang Zheng* \\ Key Laboratory of Quark and Lepton Physics (MOE) and \\ Institute of Particle Physics, Central China Normal University, \\ Wuhan 430079, China \\ E-mail: zhengleccnu.edu.cn
}

\section{E.C. Aschenauer}

Physics Department, Brookhaven National Laboratory, Upton, NY 11973, U.S.A.

E-mail: elke@bnl.gov

\section{J.H. Lee}

Physics Department, Brookhaven National Laboratory, Upton, NY 11973, U.S.A.

E-mail: jhlee@bnl.gov

\section{Bo-Wen Xiao}

Key Laboratory of Quark and Lepton Physics (MOE) and Institute of Particle Physics, Central China Normal University, Wuhan 430079, China

E-mail: xiaobowen@phy. ccnu. edu.cn

\section{Zhongbao Yin}

Key Laboratory of Quark and Lepton Physics (MOE) and Institute of Particle Physics, Central China Normal University, Wuhan 430079, China

E-mail: yinzb@phy.ccnu.edu.cn

Sivers function describes the anisotropy of parton distributions inside a transversely polarized nucleon in the momentum space. The study of the largely unexplored gluon Sivers function (GSF) is important to obtain a complete picture of the 2+1D momentum structure of nucleons. It is proposed that the GSF can be studied through dihadron single spin asymmetry (SSA) with collisions of electrons on transversely polarized protons at a future high energy, high luminosity Electron-Ion Collider (EIC). In this work, a detailed study on the feasibility of measuring the dihadron SSA arising from the GSF is presented. Due to significantly larger statistics, we find final states with charged dihadron pairs are more sensitive to GSF as compared to $K^{+} K^{-}$and $D \bar{D}$ final states.

The 26th International Nuclear Physics Conference

11-16 September, 2016

Adelaide, Australia

${ }^{*}$ Speaker. 


\section{Introduction}

In recent years, an important quest in hadron physics is to explore the multi-dimensional structure of the nucleon based on the partonic degrees of freedom. Beyond rather sophisticated studies on collinear parton distribution functions, the current forefront of hadronic structure research has led us to a three-dimensional tomographic view of the nucleon structure by including parton transverse momentum and space information. These extensions can provide unique ways of connecting underlying parton dynamics with the nucleon properties.

The transverse momentum extensions to the longitudinal parton momentum distributions can be utilized in the transverse momentum dependent (TMD) factorization framework. With the information of confined parton motion in TMD, correlations between parton transverse momentum and their spin, as well as the spin of their parent nucleon can be matched to a group of TMD parton distributions (TMDs) [1]. The Sivers function $f_{1 T}^{\perp}$ [2], which describes the azimuthally asymmetric distribution of unpolarized partons in transverse momentum space inside a transversely polarized nucleon, has received the widest attention both phenomenologically and experimentally among all TMDs.

The Sivers effect arises due to the color exchanges between the struck parton and the nucleon remnant during the initial- and final-state interactions. It was found that, due to the gauge invariance in QCD, the Sivers function is not universal in different high energy scattering processes [3]. Measuring the transverse spin phenomena related to the quark and gluon Sivers function offers an opportunity to test this process dependence deeply connected to our understanding of QCD factorization.

Experimentally, the quark Sivers function has been measured in the semi-inclusive DIS data by the HERMES, COMPASS, and JLab Hall A collaborations $[4,5,6]$ and the polarized protonproton data by the STAR and PHENIX collborations over the years. There is a prospective hint that the W boson measurement [7] at STAR favors a sign change for the Sivers function relative to that in SIDIS, which represents an important proof for the non-universality of the Sivers function. However, due to the large uncertainties and limited low $Q^{2}$ coverage of the SIDIS experimental data, only valence quark Sivers function at high $x$ can be constrained with the phenomenological studies [8]. No solid constraint can be drawn on the low $x$ or sea quark Sivers function till this moment. With the coming of the future high energy, high luminosity Electron-Ion Collider [9], the unexplored sea quark region could be ideally studied with dramatical improvement to the current data situation.

The gluon Sivers function (GSF), on the other hand, is poorly known at the current stage [10]. Fits to the Sivers asymmetry for SIDIS data favors a $30 \%$ gluon to quark Sivers ratio based on the theoretical expectation from large $N_{c}$ considerations for $x \sim 1 / N_{c}$ [8]. It has been shown in Ref. $[11,12]$ that gluon transverse momentum distribution can be mapped through quark-antiquark jet correlation in deep inelastic scattering (DIS). The open charm production in electron-proton scattering $e p^{\uparrow} \rightarrow e^{\prime} c \bar{c} X$, for instance, is supposed to be a direct probe to the gluon Sivers effect [9]. A model study has been carried out in [1] and the related experimental considerations are discussed in [13]. Besides, we can also utilize the charged $K$ meson pair or high $p_{T}$ charged dihadron pair measurements, which are more statistically favored than the charm production channels. In this work, we will illustrate the detailed information on the EIC projections for these measurements. 
Based on a TMD framework that treats the dihadron pairs in the back-to-back correlation limit, we will estimate the size of single spin asymmetry (SSA) arising from the gluon Sivers effect. Within the current design of a future EIC, we will perform a systematic study on the feasibility of measuring gluon Sivers effect with the $D^{0}$ meson pair, $K^{+} K^{-}$pair and charged dihadron pair measurements. The $D^{0}$ meson can be reconstructed through the $D^{0} \rightarrow \pi K$ decay channel. The PYTHIA Monte Carlo generator [14] has been employed to determine the event rates expected at an EIC with the lepton-proton beam energy configuration $20 \mathrm{GeV} \times 250 \mathrm{GeV}$.

\section{Single spin asymmetry (SSA) arising from the gluon Sivers effect}

The Sivers function describes the distribution of unpolarized partons with flavor $a$ inside a transversely polarized prton with mass $M_{p}$ and can be expressed in a way following the Trento convention in Ref. [15]:

$$
\hat{f}_{a / p^{\uparrow}}\left(x, k_{\perp}\right)=f_{a / p}\left(x, k_{\perp}\right)-f_{1 T}^{\perp a}\left(x, k_{\perp}\right) \frac{\vec{S} \cdot\left(\hat{\vec{P}} \times \vec{k}_{\perp}\right)}{M_{p}} .
$$

The first term provides an axially symmetric contribution from the unpolarized parton distribution, while the second term containing $f_{1 T}^{\perp a}\left(x, k_{\perp}\right)$ generates a distortion away from the center in the $\vec{k}_{\perp}$ plane with respect to the proton spin direction dependent on light-front momentum fraction $x$ and parton initial transverse momentum $\vec{k}_{\perp} . \vec{P}$ and $\vec{S}$ represent the momentum and the normalized transverse spin vector of the proton, respectively.

The production of high transverse momenta hadron pairs in DIS through $\gamma^{*} g \rightarrow q \bar{q}$ is sensitive to the gluon contributions, although it may have small contribution from the quark channel. If $k_{1}$ and $k_{2}$ are the four momenta of the outgoing quarks, one can obtain the dihadron cross section as a generalization to the unpolarized case in [16] with the transverse momentum imbalance $k_{\perp}=$ $\left|\vec{k}_{1 \perp}+\vec{k}_{2 \perp}\right|$ and the dijet transverse momentum scale $P_{\perp}=\left|\vec{k}_{1 \perp}-\vec{k}_{2 \perp}\right| / 2$ :

$$
\begin{aligned}
\frac{d \sigma_{\text {tot }}^{\gamma^{*}+p^{\uparrow} \rightarrow h_{1}+h_{2}+X}}{d z_{h 1} d z_{h 2} d^{2} p_{h 1 \perp} d^{2} p_{h 2 \perp}}= & \quad C \int_{z_{h 1}}^{1-z_{h 2}} \sum_{q} d z_{q} \frac{z_{q}\left(1-z_{q}\right)}{z_{h 2}^{2} z_{h 1}} d^{2} p_{1 \perp} d^{2} p_{2 \perp} \hat{f}_{g / p^{\uparrow}}\left(x_{g}, k_{\perp}\right) \\
& \times H_{\text {tot }}^{\gamma^{*} g \rightarrow q \bar{q}}\left(z_{q}, k_{1 \perp}, k_{2 \perp}\right) e_{q}^{2} D_{h 1 / q}\left(\frac{z_{h 1}}{z_{q}}, p_{1 \perp}\right) D_{h 2 / \bar{q}}\left(\frac{z_{h 2}}{1-z_{q}}, p_{2 \perp}\right),
\end{aligned}
$$

where $C$ gives the normalization factor, $z_{q}$ is the momentum fraction of produced quark $q$ with respect to the incoming virtual photon and $H_{t o t}^{\gamma^{*} g \rightarrow q \bar{q}}\left(z_{q}, k_{1 \perp}, k_{2 \perp}\right)$ gives the combined hard factor that incorporates both longitudinal and transverse component of the virtual photon. In our analysis, we restrict ourselves to a kinematic configuration with $k_{\perp} \ll P_{\perp}$ known as the correlation limit [17].

By choosing the reference frame in which proton beam with momentum $\vec{P}$ is moving in $z$ direction and is transversely polarized along $y$ axis, one can come to an explicit form of the mixed vector product in Eq. 2.1 as $\vec{S} \cdot\left(\hat{\vec{P}} \times \vec{k}_{\perp}\right)=k_{\perp} \cos \left(\phi_{k}\right)$ with $\phi_{k}$ being the azimuthal angle of $\vec{k}_{\perp}$. A factorized Gaussian parameterization has been adopted for the transverse momentum dependent unpolarized parton distribution function $f_{g / p}\left(x_{g}, k_{\perp}\right)=f_{g / p}\left(x_{g}\right) \frac{e^{-k_{\perp}^{2} /<k_{\perp}^{2}>}}{\pi\left\langle k_{\perp}^{2}\right\rangle}$ with $\left\langle k_{\perp}^{2}\right\rangle=0.25 \mathrm{GeV}^{2}$ and fragmentation function $D\left(z, p_{\perp}\right)=D(z) \frac{e^{-p_{\perp}^{2} /\left\langle p_{\perp}^{2}\right\rangle}}{\pi\left\langle p_{\perp}^{2}\right\rangle}$ with $\left\langle p_{\perp}^{2}\right\rangle=0.2 \mathrm{GeV}^{2}$.

There exists to be a strong correlation between the kinematics of a selected hadron and its quark parent. Therefore, one can use the measurable hadron variables $P_{T}^{\prime}=\left|p_{h 1 \perp}-p_{h 2 \perp}\right| / 2$ and 
$k_{T}^{\prime}=\left|p_{h 1 \perp}+p_{h 2 \perp}\right|$ to access the underlying parton kinematic variables $P_{\perp}$ and $k_{\perp}$. The GSF can be studied in the dihadron single spin asymmetry (SSA) with the notation of $\Delta^{N} f_{a / p^{\uparrow}}\left(x, k_{\perp}\right)=$ $-\frac{2 k_{\perp}}{M_{p}} f_{1 T}^{\perp a}\left(x, k_{\perp}\right)$ as follows:

$$
A_{U T}\left(\phi_{S k^{\prime}}, k_{T}^{\prime}\right)=\frac{d \sigma^{\uparrow}\left(\phi_{S k^{\prime}}, k_{T}^{\prime}\right)-d \sigma^{\downarrow}\left(\phi_{S k^{\prime}}, k_{T}^{\prime}\right)}{d \sigma^{\uparrow}\left(\phi_{S k^{\prime}}, k_{T}^{\prime}\right)+d \sigma^{\downarrow}\left(\phi_{S k^{\prime}}, k_{T}^{\prime}\right)} \propto \frac{\Delta^{N} f_{g / p^{\uparrow}}\left(x, k_{\perp}\right)}{f_{1}^{g}\left(x_{g}, k_{\perp}\right)},
$$

where subscript " $\mathrm{U}$ " represents the unpolarized electrion beam and " $\mathrm{T}$ " indicates the proton beam is transversely polarized. $\phi_{S k^{\prime}}=\phi_{S}-\phi_{k_{T}^{\prime}}$ indicates the angular difference between total dihadron transverse momentum $\vec{k}_{T}^{\prime}$ and the polarized proton spin direction $\vec{S}_{\perp}$. The size of SSA is proportional to the corresponding Sivers function divided by the unpolarized parton distributions.

We provide an estimate to the possible size of single spin asymmetry in the following studies with three model assumptions. The maximized one can be obtained with an ansatz which saturates the positivity bound $f_{1 T}^{\perp g}=-\frac{2 \sigma M_{p}}{k_{\perp}^{2}+\sigma^{2}} f_{g}\left(x, k_{\perp}\right), \sigma=0.8 \mathrm{GeV}$. An intermediate size gluon Sivers function has been set with $5 \%$ level of the positivity bound. In the end, a minimized set of gluon Sivers parameterization has been taken from the SIDIS1 fits to the $\pi^{0} A_{N}$ data in pp collisions at RHIC [18]. The corresponding Sivers functional form for quark is taken from [19].

\section{Monte Carlo simulation setup}

In this section, we will describe the setup of our Monte Carlo simulation tool for the event generations. We use the PYTHIA-6.4 Monte Carlo program to give a projection on the specific dihadron production event rates that could be expected at an EIC.

The size of the asymmetry can be obtained within the framework discussed in Sec. 2. If the number of dihadron pairs reconstructed in a kinematic bin is $N$, one can estimate the statistical uncertainty of the SSA in that bin as $\left(\delta A_{U T}\right)^{2}=\frac{1}{P^{2} N}-\frac{A_{U T}^{2}}{N}$ [20]. In the following studies, error bars are obtained assuming a polarization $P=70 \%$. All the simulations done in this work for the EIC energy scale has been set up with the electron and proton beam energy configuration of $20 \times$ $250 \mathrm{GeV}$.

Back-to-back dihadron pairs selected by our requirements are supposed to be produced mostly in the Photon-Gluon Fusion (PGF) $\gamma^{*} g \rightarrow q \bar{q}$ process. Noticing that the quark initiated process QCD Compton (QCDC) $\gamma^{*} q \rightarrow q g$ contribution can not be disentangled from PGF process in $K^{+} K^{-}$ and high $p_{T}$ charged dihadron pair measurement, we will take into account all the information on the quark Sivers effect. The final SSA can be expressed as a weighted sum of those from gluon and quark initiated process: $A_{U T}=w^{P G F} A_{U T}^{P G F}+w^{Q C D C} A_{U T}^{Q C D C}$, in which $w^{P G F}$ and $w^{Q C D C}$ represents the event fraction of PGF and QCDC process in the event sample, respectively. The quark initiated dihadron cross section can be obtained similarly as Eq. 2.2 with the a combination of different quark flavors.

\section{Results and discussions}

The laboratory coordinate system has been defined with a convention the proton beam moving in $+z$ and the incoming electron along $-z$. The event kinematics has been constrained to $0.01<$ 
$y<0.95$ and $1 \mathrm{GeV}^{2}<Q^{2}<20 \mathrm{GeV}^{2}$. The selection of this kinematic range delivers the average event properties as $\left\langle x_{B j}\right\rangle=0.0012,\left\langle Q^{2}\right\rangle=2.5 \mathrm{GeV}^{2},\langle W\rangle=54.6 \mathrm{GeV}$. In the following studies, we will explore the feasibility of measuring the gluon Sivers effect with the selected final states of $D \bar{D}$ pair, $K^{+} K^{-}$pair and charged dihadron pair.

\section{1 gluon SSA with $D \bar{D}$ pair}

The $D^{0} \rightarrow \pi K$ decay channel is used in this analysis by taking the advantage of its large branching ratio $3.87 \%$ and the capability of identifying its decay vertex with the vertex tracking detector at an EIC. Decay products momenta are typically a few $\mathrm{GeV}$ in the most central region, but extend to $10 \mathrm{GeV}$ in the region away from central.

The $D$ meson decay products are not allowed to exceed the acceptance range region $\left|\eta_{\text {Lab }}^{\pi / K}\right|<$ 3.5. To guarantee the decay products of $D^{0}$ can reach time projection chamber for particle identification, a transverse momenta cut $p_{T L a b}^{\pi / K}>0.2 \mathrm{GeV}$ has been applied to the decay products. As the kinematics of a directly generated $D^{0}$ is of little difference compared to that of a heavier $D$ meson state generating it, we include all the $D^{0}$ in our study. To select a pair of back-to-back particles in the correlation limit, we make a cut on the reconstructed $D^{0}$ and $\bar{D}^{0}$ pairs: $k_{T}^{\prime}<0.7 P_{T}^{\prime} \cdot z_{h}>0.1$ has been applied to the decay products to eliminate contributions from the remnant fragmentations. Meanwhile, it is also found that the feed-down correction is not significant for the analysis. The kinematics of $D^{0}$ meson from $D^{*}$ is not significantly different from the directly generated $D^{0}$ mesons.

For the $D^{0}$ meson production, quark subscript in Eq. 2.2 represents charm quark as $q=c$. The hard factor $H_{t o t}^{\gamma^{*} g \rightarrow c \bar{c}}$ is given by Ref. [17]. The fragmentation function $D(z) \propto z^{\alpha}(1-z)^{\beta} e^{\gamma z(1-z)}$ with $\alpha=2.86, \beta=1.57, \gamma=5.66$, which fairly describes the $D^{0}$ spectrum in $e^{+} e^{-}$annihilation, has been applied to estimate the $D^{0}$ meson pair cross section. As suggested by Ref. [21], the gluon momentum fraction $x_{g}$ can be determined as $x_{g}=\frac{Q^{2}}{s}+\frac{\left(P_{\perp}^{2}+m_{c}^{2}\right)}{z_{q}\left(1-z_{q}\right) s}$, where charm quark mass $m_{c}=1.3 \mathrm{GeV}$ and $s$ gives the center of mass energy for the $\gamma^{*} p$ system.

With the $D \bar{D}$ pair production in the final state, the quark initiated process is significantly suppressed that one can have a clean probe to the gluon Sivers effect. The sensitivity of this measurement to the possible gluon Sivers size has been shown in Fig. 1. The statistical uncertainty is obtained with an assumption of the integrated luminosity $\mathscr{L}_{\text {int }}=20 \mathrm{fb}^{-1}$.

\section{2 gluon SSA with high $p_{T}$ charged dihadron pair and $K^{+} K^{-}$pair}

The gluon dynamics can also be accessed with the light flavored dihadron pair probes. To estimate these final states, DSS fragmentation has been utilized in the numerical estimations for the signal size. These processes will suffer the contamination in SSA from quark Sivers effects. We can obtain the fraction of quark and gluon initiated process $w^{P G F}$ and $w^{Q C D C}$ in PYTHIA simulations.

A high $p_{T}$ cut for the dihadron pair is usually needed to minimize the quark channel contaminations. It can be found in Fig. 2 that the quark initiated process $\gamma^{*} q \rightarrow q$ and $\gamma^{*} q \rightarrow g q$ will be suppressed with maximized $\gamma^{*} g \rightarrow q \bar{q}$ fraction by applying a transverse momentum cut $p_{T}>1.7$ $\mathrm{GeV}$. The acceptance for the charged dihadron has been designed to be $\left|\eta_{L a b}\right|<4.5$. The correlated dihadron pairs are selected again with the correlation limit requirement $k_{T}^{\prime}<0.7 P_{T}^{\prime}$. The energy 


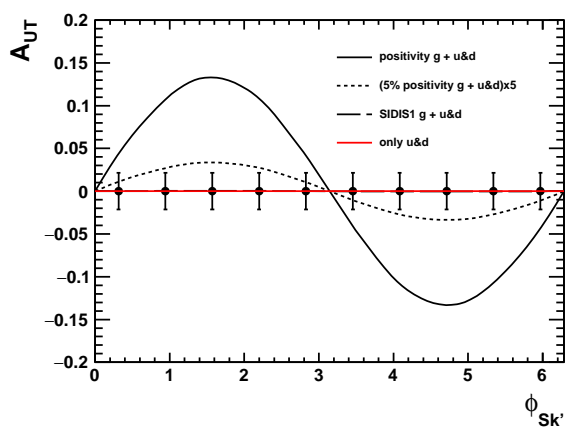

Figure 1: (Color online) Projection on the $D \bar{D}$ pair single spin asymmetry dependence varying with $\phi_{S k^{\prime}}$ shown with different gluon Sivers magnitude. Vertical bar represents the statistical error obtained assuming the kinematic cut $\left|\eta_{\text {Lab }}^{\pi / K}\right|<3.5, p_{T L a b}^{\pi / K}>0.2 \mathrm{GeV}, z^{\pi / K}>0.1, k_{T}^{\prime}<0.7 P_{T}^{\prime}, 0.01<y<0.95$ and $1 \mathrm{GeV}^{2}<$ $Q^{2}<20 \mathrm{GeV}^{2}$ at the electron-proton beam energy $20 \mathrm{GeV} \times 250 \mathrm{GeV}$, if the total luminosity $\mathscr{L}_{\text {int }}=20 \mathrm{fb}^{-1}$.

fraction of the involved particles taken from the exchanged virthal photon must be $z_{h}>0.1$. The corresponding estimation of the projected statistical resolution has been shown in Fig. 3 if the total statistics amounts to $\mathscr{L}_{\text {int }}=20 \mathrm{fb}^{-1}$.

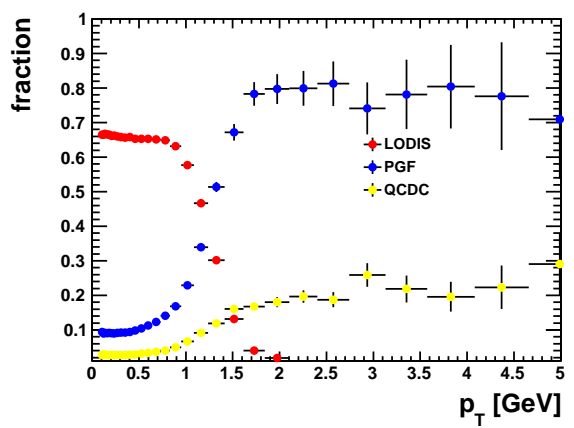

Figure 2: (Color online) Fraction of the charged particle cross section out of the total cross section versus transverse momentum from different processes.

The selection of $K^{+} K^{-}$pair is employed to maximize the $\gamma^{*} g \rightarrow s \bar{s}$ contribution, which is more favored for the gluon dynamics study. The identification of charged kaon has been assumed to be applicable only within the range of $\left|\eta_{L a b}\right|<3.5$. With all the experimental considerations, the projected statistical uncertainty compared to the expected signal size can be found in Fig. 4 assuming an integrated luminosity $\mathscr{L}_{\text {int }}=20 \mathrm{fb}^{-1}$.

\subsection{Discussions}

By confronting the expected signal size of all three possible probes discussed above and the EIC projections, one can compare the feasibility and signal sensitivity of these measurements to gluon Sivers effect with a variation of statistics assumption. The gluon Sivers sensitivity largely relies on the fraction of gluon channels contributing to the measurement. The detailed gluon channel fraction can be collected as $w_{D \bar{D}}^{P G F}=99.4 \%, w_{K^{+} K^{-}}^{P G F}=92.7 \%, w_{\text {dihadron }}^{P G F}=80.0 \%$ from PYTHIA simulation. Based on the kinematic cuts and binning method discussed in the last section, we 


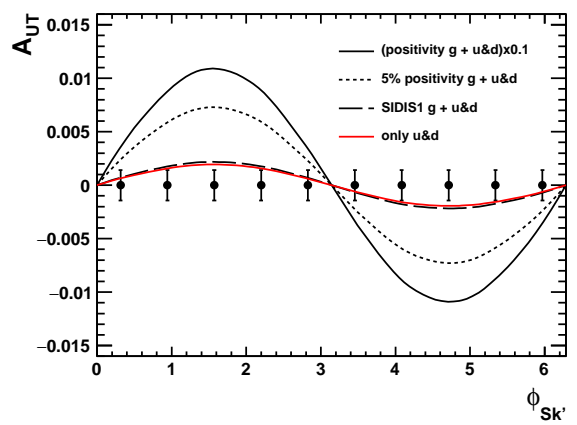

Figure 3: (Color online) Projection on the charged dihadron pair single spin asymmetry dependence varying with $\phi_{S k^{\prime}}$ shown with different gluon Sivers magnitude. Vertical bar represents the statistical error obtained assuming the kinematic cut $\left|\eta_{\text {Lab }}^{h}\right|<4.5, p_{T}^{h}>1.7 \mathrm{GeV}, z^{h}>0.1, k_{T}^{\prime}<0.7 P_{T}^{\prime}, 0.01<y<0.95$ and $1 \mathrm{GeV}^{2}<$ $Q^{2}<20 \mathrm{GeV}^{2}$ at the electron-proton beam energy $20 \mathrm{GeV} \times 250 \mathrm{GeV}$, if the total luminosity $\mathscr{L}_{\text {int }}=20 \mathrm{fb}^{-1}$.

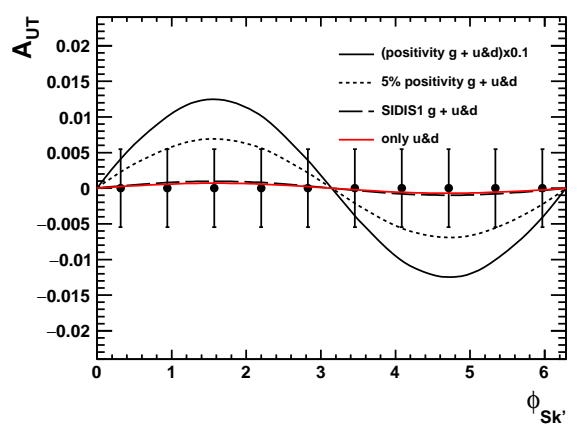

Figure 4: (Color online) Projection on the $K^{+} K^{-}$pair single spin asymmetry dependence varying with $\phi_{S k^{\prime}}$ shown with different gluon Sivers magnitude Vertical bar represents the statistical error obtained assuming the kinematic cut $\left|\eta_{\text {Lab }}^{K}\right|<3.5, p_{T}^{K}>1.7 \mathrm{GeV}, z^{K}>0.1, k_{T}^{\prime}<0.7 P_{T}^{\prime}, 0.01<y<0.95$ and $1 \mathrm{GeV}^{2}<Q^{2}<$ $20 \mathrm{GeV}^{2}$ at the electron-proton beam energy $20 \mathrm{GeV} \times 250 \mathrm{GeV}$, if the total luminosity $\mathscr{L}_{\text {int }}=20 \mathrm{fb}^{-1}$.

can obtain the statistical error of the single spin asymmetry ordering with different probes as $\delta A_{U T}^{D \bar{D}} \sim 3.66 \delta A_{U T}^{K^{+} K^{-}} \sim 14.71 \delta A_{U T}^{\text {dihadron }}$.

\section{Summary}

In this work, we have performed a calculation on the magnitude of SSA for $D \bar{D}, K^{+} K^{-}$and charged dihadron pair production arising from the gluon Sivers effect. Expectations from different gluon Sivers models are obtained in a transverse momentum dependent factorization framework. Detailed experimental considerations are considered study the possible feasibilities of these measurements. It is found that the $D \bar{D}$ meson pair probe is less affected by the quark initiated process, but the $K^{+} K^{-}$and charged dihadron pair probes are more favored in terms of the statistics.

This measurement will largely improve our understanding to the gluon Sivers effect. Due to the different gauge links involved in the processes, the gluon Sivers effect extracted from electronproton collisions would be an independent gluon Sivers function distinct from that obtained in 
proton-proton collisions and can hardly be accessed in other collisions. Thus, gluon Sivers studies at an EIC will provide a unique test of the fundamental non-perturbative QCD effects.

\section{References}

[1] D. Boer et al., (2011), 1108.1713.

[2] D. W. Sivers, Phys. Rev. D41, 83 (1990).

[3] A. V. Efremov, K. Goeke, S. Menzel, A. Metz, and P. Schweitzer, Phys. Lett. B612, 233 (2005), hep-ph/0412353.

[4] HERMES, A. Airapetian et al., Phys. Rev. Lett. 94, 012002 (2005), hep-ex/0408013.

[5] COMPASS, M. Alekseev et al., Phys. Lett. B673, 127 (2009), 0802.2160.

[6] Jefferson Lab Hall A, X. Qian et al., Phys. Rev. Lett. 107, 072003 (2011), 1106.0363.

[7] STAR, L. Adamczyk et al., Phys. Rev. Lett. 116, 132301 (2016), 1511.06003.

[8] M. Anselmino et al., Eur. Phys. J. A39, 89 (2009), 0805.2677.

[9] A. Accardi et al., (2012), 1212.1701.

[10] D. Boer, C. LorcÃl', C. Pisano, and J. Zhou, Adv. High Energy Phys. 2015, 371396 (2015), 1504.04332.

[11] F. Dominguez, B.-W. Xiao, and F. Yuan, Phys. Rev. Lett. 106, 022301 (2011), 1009.2141.

[12] D. Boer, S. J. Brodsky, P. J. Mulders, and C. Pisano, Phys. Rev. Lett. 106, 132001 (2011), 1011.4225.

[13] T. Burton, Gluon sivers and experimental considerations for TMDs, in Proceedings, 20th International Workshop on Deep-Inelastic Scattering and Related Subjects (DIS 2012), pp. 377-380, 2012, 1212.3590, [,377(2012)].

[14] T. Sjostrand, S. Mrenna, and P. Z. Skands, JHEP 05, 026 (2006), hep-ph/0603175.

[15] A. Bacchetta, U. D’Alesio, M. Diehl, and C. A. Miller, Phys. Rev. D70, 117504 (2004), hep-ph/0410050.

[16] L. Zheng, E. C. Aschenauer, J. H. Lee, and B.-W. Xiao, Phys. Rev. D89, 074037 (2014), 1403.2413.

[17] F. Dominguez, C. Marquet, B.-W. Xiao, and F. Yuan, Phys. Rev. D83, 105005 (2011), 1101.0715.

[18] U. D’Alesio, F. Murgia, and C. Pisano, JHEP 09, 119 (2015), 1506.03078.

[19] M. Anselmino et al., Sivers Distribution Functions and the Latest SIDIS Data, in 19th International Workshop on Deep-Inelastic Scattering and Related Subjects (DIS 2011) Newport News, Virginia, April 11-15, 2011, 2011, 1107.4446.

[20] G. Bunce, N. Saito, J. Soffer, and W. Vogelsang, Ann. Rev. Nucl. Part. Sci. 50, 525 (2000), hep-ph/0007218.

[21] M. Anselmino, M. Boglione, U. D’Alesio, E. Leader, and F. Murgia, Phys. Rev. D70, 074025 (2004), hep-ph/0407100. 